Article

\title{
Some Bounds on Eigenvalues of the Hadamard Product and the Fan Product of Matrices
}

\author{
Qianping Guo ${ }^{1, * \mathbb{C}}$, Jinsong Leng ${ }^{2, *}$, Houbiao $\mathrm{Li}^{2}{ }^{-}$and Carlo Cattani ${ }^{3}$ \\ 1 Department of Mathematics and Information Science, Henan University of Finance and Economics, \\ Zhengzhou 450046, China \\ 2 School of Mathematical Sciences, University of Electronic Science and Technology of China, \\ Chengdu 611731, China; lihoubiao0189@163.com \\ 3 Engineering School, DEIM, University of Tuscia, 01100 Viterbo, Italy; cattani@unitus.it \\ * Correspondence: guoqianpinglei@163.com (Q.G.); jinsongleng@126.com (J.L.); Tel.: +86-135-5002-8157 (J.L.)
}

Received: 14 November 2018; Accepted: 31 January 2019; Published: 3 February 2019

check for updates

\begin{abstract}
In this paper, an upper bound on the spectral radius $\rho(A \circ B)$ for the Hadamard product of two nonnegative matrices $(A$ and $B)$ and the minimum eigenvalue $\tau(C \star D)$ of the Fan product of two $M$-matrices $(C$ and $D)$ are researched. These bounds complement some corresponding results on the simple type bounds. In addition, a new lower bound on the minimum eigenvalue of the Fan product of several $M$-matrices is also presented. These results and numerical examples show that the new bounds improve some existing results.
\end{abstract}

Keywords: Hadamard product; nonnegative matrices; spectral radius; fan product; $M$-matrix; inverse $M$-matrix; minimum eigenvalue

\section{Introduction}

As is well known, the Hadamard, Fan and Kronecker products play an important role in matrix methods for statistics and econometrics [1,2]. The research on eigenvalues of the Hadamard and Fan products of matrices is always one of the hot topics in matrix theory see References [3-16]. In this paper, we will continue to research this topic and present some new results.

First, we introduce some notations, see Reference [17]. Throughout this article, a positive integer $n, N$ denotes the set $\{1,2, \cdots, n\}$. The sets of all $n \times n$ real and complex matrices are denoted by $\mathbb{R}^{n \times n}$ and $\mathbb{C}^{n \times n}$, respectively. For two real $n \times n$ matrices $A=\left(a_{i j}\right)$ and $B=\left(b_{i j}\right)$, we write $A \geq B$ $(A>B)$ if $a_{i j} \geq b_{i j}\left(a_{i j}>b_{i j}\right)$ for all $i, j \in N$. If $A \geq 0(A>0)$, we say that $A$ is a nonnegative (positive) matrix. $A^{T}$ means the transposition of matrix $A$. The spectral radius of $A$ is denoted by $\rho(A)$. If $A$ is a nonnegative matrix, the Perron-Frobenius theorem guarantees that $\rho(A) \in \sigma(A)$, where $\sigma(A)$ is the set of all eigenvalues of $A$. Moreover, a matrix $A$ is called reducible if there exists a nonempty proper subset $I \subset N$ such that $a_{i j}=0, \forall i \in I, \forall j \notin I$. If $A$ is not reducible, then we call $A$ irreducible (see Reference [7]).

In addition, we denote by $\mathcal{Z}_{n}$ the class of all $n \times n$ real matrices of whose off-diagonal entries are non-positive. If $A \in \mathcal{Z}_{n}$, then the minimum eigenvalue of $A$ is defined by $\tau(A):=\min \{\operatorname{Re}(\lambda) \mid \lambda \in$ $\sigma(A)\}$. As a special case of $\mathcal{Z}_{n}$, a matrix $A=\left(a_{i j}\right) \in \mathcal{Z}_{n}$ is called a nonsingular $M$-matrix if $A$ is nonsingular and $A^{-1} \geq 0$ (see Reference [8]). $\mathcal{M}_{n}$ denotes the set of all nonsingular $M$-matrices. Generally speaking, the following simple facts are well known (see Problems 16, 19, and 28 in Section 2.5 of Reference [8]):

1. If $A \in \mathcal{Z}_{n}$, then $\tau(A) \in \sigma(A)$;

2. If $A, B \in \mathcal{M}_{n}$, and $A \geq B$, then $\tau(A) \geq \tau(B)$, moreover, $\tau(A) \leq \min \left\{a_{i i}\right\}$; 
3. If $A \in \mathcal{M}_{n}$, then there exists a positive eigenvalue of $A$ equal to $\tau(A)=\left[\rho\left(A^{-1}\right)\right]^{-1}$, where $\rho\left(A^{-1}\right)$ is the Perron eigenvalue of the nonnegative matrix $A^{-1}$.

Finally, let $A$ be an irreducible nonsingular $M$-matrix. It is well known that there exist positive vectors $u$ and $v$ such that $A u=\tau(A) u$ and $v^{T} A=\tau(A) v^{T}$, where $u$ and $v$ are right and left Perron eigenvectors of $A$, respectively. The Hadamard product of $A=\left(a_{i j}\right) \in \mathbb{C}^{n \times n}$ and $B=\left(b_{i j}\right) \in \mathbb{C}^{n \times n}$ is defined by $A \circ B=\left(a_{i j} b_{i j}\right) \in \mathbb{C}^{n \times n}$. As a variant of the Hadamard product, the Fan product of two real matrices $A, B \in \mathcal{Z}_{n}$ is denoted by $A \star B=C=\left(c_{i j}\right) \in \mathcal{Z}_{n}$, where

$$
c_{i j}= \begin{cases}-a_{i j} b_{i j}, & \text { if } i \neq j, \\ a_{i i} b_{i i}, & \text { if } i=j .\end{cases}
$$

\section{A Mixed Type Bound for Eigenvalues of the Hadamard and Fan Products of Two Matrices}

In recent years, on the problem of $\rho(A \circ B)$ of two nonnegative matrices $A$ and $B$, there exist some rich results based on the $\rho(A)$ and $\rho(B)$.

- In Reference [8], p. 358, there is an inequality for $\rho(A \circ B)$ : if $A, B \in \mathbb{R}^{n \times n}(A \geq 0, B \geq 0)$, then $\rho(A \circ B) \leq \rho(A) \rho(B)$. According to Exercise (Reference [8], p. 358), we know this inequality can be very weak in some cases. For example, if $A=I, B=J$, the matrix of all ones. Then

$$
\rho(A \circ B)=\rho(A) \ll \rho(A) \rho(B)=n
$$

when $n$ is very large. Obviously, the equality can occur when $A=B=I$.

- Fang [4] gave an upper bound for $\rho(A \circ B)$, i.e.,

$$
\rho(A \circ B) \leq \max _{1 \leq i \leq n}\left\{2 a_{i i} b_{i i}+\rho(A) \rho(B)-b_{i i} \rho(A)-a_{i i} \rho(B)\right\}
$$

which is sharper than the bound $\rho(A) \rho(B)$ in Reference [8], p. 358.

- Liu et al. [14] further improved the above results and obtained the following bound:

$$
\begin{aligned}
\rho(A \circ B) & \leq \max _{i \neq j} \frac{1}{2}\left\{a_{i i} b_{i i}+a_{j j} b_{j j}+\left[\left(a_{i i} b_{i i}-a_{j j} b_{j j}\right)^{2}\right.\right. \\
& \left.\left.+4\left(\rho(A)-a_{i i}\right)\left(\rho(B)-b_{i i}\right)\left(\rho(A)-a_{j j}\right)\left(\rho(B)-b_{j j}\right)\right]^{\frac{1}{2}}\right\} .
\end{aligned}
$$

Cheng [3] also obtained the following results based on the row maximum non-diagonal elements and the commutative property of Hadamard product.

Theorem 1. Let $A=\left(a_{i j}\right)$ and $B=\left(b_{i j}\right)$ be nonnegative matrices, $s_{i}=\max _{j \neq i}\left\{a_{i j}\right\}, t_{i}=\max _{j \neq i}\left\{b_{i j}\right\}$, then

$$
\begin{aligned}
& \rho(A \circ B) \leq \\
& \min \left\{\begin{array}{l}
\max _{i \neq j} \frac{1}{2}\left\{a_{i i} b_{i i}+a_{j j} b_{j j}+\left[\left(a_{i i} b_{i i}-a_{j j} b_{j j}\right)^{2}+4 t_{i} t_{j}\left(\rho(A)-a_{i i}\right)\left(\rho(A)-a_{j j}\right)\right]^{1 / 2}\right\}, \\
\max _{i \neq j} \frac{1}{2}\left\{a_{i i} b_{i i}+a_{j j} b_{j j}+\left[\left(a_{i i} b_{i i}-a_{j j} b_{j j}\right)^{2}+4 s_{i} s_{j}\left(\rho(B)-b_{i i}\right)\left(\rho(B)-b_{j j}\right)\right]^{1 / 2}\right\} .
\end{array}\right\} .
\end{aligned}
$$

For the reader's convenience and our results equivalence, we call Equation (3) a 'simple' type bound.

Motivated by the work of Fang $[3,4,14]$, in this section, we continue this work on the upper bound on $\rho(A \circ B)$ of two nonnegative matrices and get a new upper bound, which improves the above existing bounds. Moreover, we similarly extend our result to the Fan product of two $M$ - matrices.

Before obtaining our results, we give some lemmas. 
Lemma 1 (Perron-Frobenius theorem [8]). If $A$ is an irreducible nonnegative matrix, there exists a positive vector $u$, such that $A u=\rho(A) u$.

Lemma 2 ([8]). If $A, B \in \mathbb{C}^{n \times n}, D$ and $E$ are positive diagonal matrices, then

$$
D(A \circ B) E=(D A E) \circ B=(D A) \circ(B E)=(A E) \circ(D B)=A \circ(D B E) .
$$

Lemma 3 (Brauer's theorem). Let $A=\left(a_{i j}\right) \in \mathbb{C}^{n \times n}(n \geq 2)$, then

$$
\sigma(A) \subset \bigcup_{i, j=1 ; i \neq j}^{n}\left\{z \in \mathbb{C}:\left|z-a_{i i}\right|\left|z-a_{j j}\right| \leq\left(\sum_{k \neq i}\left|a_{i k}\right|\right)\left(\sum_{k \neq j}\left|a_{j k}\right|\right)\right\} .
$$

Let $A=\left(a_{i j}\right) \in \mathbb{C}^{n \times n}(n \geq 2)$, and $d_{1}, d_{2}, \cdots, d_{n}>0$. Denote $D=\operatorname{diag}\left(d_{1}, d_{2}, \cdots, d_{n}\right)$, since $D^{-1} A D$ and $A$ have the same eigenvalues, then by Lemma 3, we obtain that all the eigenvalues of $A$ lie in the region

$$
\bigcup_{i, j=1 ; i \neq j}^{n}\left\{z \in \mathbb{C}:\left|z-a_{i i}\right|\left|z-a_{j j}\right| \leq\left(\sum_{k \neq i} \frac{d_{k}}{d_{i}}\left|a_{i k}\right|\right)\left(\sum_{k \neq j} \frac{d_{k}}{d_{j}}\left|a_{j k}\right|\right)\right\} .
$$

Now, we present the main theorem of this section. Here, we call it a 'mixed' type.

Theorem 2. Let $A=\left(a_{i j}\right)$ and $B=\left(b_{i j}\right)$ be nonnegative matrices, $s_{i}=\max _{j \neq i}\left\{a_{i j}\right\}, t_{i}=\max _{j \neq i}\left\{b_{i j}\right\}$, then

$$
\begin{aligned}
\rho(A \circ B) \leq & \max _{i \neq j} \frac{1}{2}\left\{a_{i i} b_{i i}+a_{j j} b_{j j}+\left[\left(a_{i i} b_{i i}-a_{j j} b_{j j}\right)^{2}\right.\right. \\
& \left.\left.+4 t_{i} s_{j}\left(\rho(A)-a_{i i}\right)\left(\rho(B)-b_{j j}\right)\right]^{\frac{1}{2}}\right\} .
\end{aligned}
$$

Proof. It is evident that the inequality (6) holds with the equality for $n=1$. Therefore, we assume that $n \geq 2$ and divide two cases to prove this problem.

Case 1. Suppose that $A \circ B$ is irreducible. Obviously, $A$ and $B$ are also irreducible. By the Perron-Frobenius theorem of the Lemma (1), there exists a positive vector $u=\left(u_{1}, u_{2}, \cdots, u_{n}\right)^{T}$ for any $D=\operatorname{diag}\left(d_{1}, d_{2}, \cdots, d_{n}\right), d_{i}>0$ such that

$$
\left(D^{-1} A D\right) u=\rho\left(D^{-1} A D\right) u=\rho(A) u,
$$

i.e.,

$$
\sum_{j \neq i} \frac{a_{i j} d_{j} u_{j}}{d_{i} u_{i}}=\rho(A)-a_{i i} .
$$

Obviously, for the matrix $B$, we obtain similarly that

$$
\sum_{j \neq i} \frac{b_{i j} d_{j} u_{j}}{d_{i} u_{i}}=\rho(B)-b_{i i} .
$$

Define $U=\operatorname{diag}\left(u_{1}, u_{2}, \cdots, u_{n}\right), C=(D U)^{-1} A(D U)$, then we see that

$$
C=\left(\begin{array}{cccc}
a_{11} & \frac{d_{2} u_{2}}{d_{1} u_{1}} a_{12} & \cdots & \frac{d_{n} u_{n}}{d_{1} u_{1}} a_{1 n} \\
\frac{d_{1} u_{1}}{d_{2} u_{2}} a_{21} & a_{22} & \cdots & \frac{d_{n} u_{n}}{d_{2} u_{2}} a_{2 n} \\
\vdots & \vdots & \ddots & \vdots \\
\frac{d_{1} u_{1}}{d_{n} u_{n}} a_{n 1} & \frac{d_{2} u_{2}}{d_{n} u_{n}} a_{n 2} & \cdots & a_{n n}
\end{array}\right)
$$


is an irreducible nonnegative matrix and

$$
C \circ B=\left(m_{i j}\right)=\left(\begin{array}{cccc}
a_{11} b_{11} & \frac{d_{2} u_{2}}{d_{1} u_{1}} a_{12} b_{12} & \cdots & \frac{d_{n} u_{n}}{d_{1} u_{1}} a_{1 n} b_{1 n} \\
\frac{d_{1} u_{1}}{d_{2} u_{2}} a_{21} b_{21} & a_{22} b_{22} & \cdots & \frac{d_{n} u_{n}}{d_{2} u_{2}} a_{2 n} b_{2 n} \\
\vdots & \vdots & \ddots & \vdots \\
\frac{d_{1} u_{1}}{d_{n} u_{n}} a_{n 1} b_{n 1} & \frac{d_{2} u_{2}}{d_{n} u_{n}} a_{n 2} b_{n 2} & \cdots & a_{n n} b_{n n}
\end{array}\right) .
$$

From Lemma 2, it is easy to have $(D U)^{-1}(A \circ B)(D U)=(D U)^{-1} A(D U) \circ B=C \circ B$, therefore, $\rho(A \circ B)=\rho(C \circ B)$.

From the inequality (4) and $\rho(A \circ B) \geq a_{i i} b_{i i}$ (see Reference [17]), for any $j \neq i \in N$, we have

$$
\begin{aligned}
\left.\left(\rho(A \circ B)-a_{i i} b_{i i}\right)(\rho(A \circ B))-a_{j j} b_{j j}\right) & \leq \sum_{k \neq i}\left|m_{i k}\right| \sum_{l \neq j}\left|m_{j l}\right| \\
& =\sum_{k \neq i} \frac{d_{k} u_{k} a_{i k} b_{i k}}{d_{i} u_{i}} \sum_{l \neq j} \frac{d_{l} u_{l} a_{j l} b_{j l}}{d_{j} u_{j}} \\
& \leq\left(\max _{k \neq i}\left\{b_{i k}\right\} \sum_{k \neq i} \frac{d_{k} u_{k} a_{i k}}{d_{i} u_{i}}\right)\left(\max _{l \neq j}\left\{a_{j l}\right\} \sum_{l \neq j} \frac{d_{l} u_{l} b_{j l}}{d_{j} u_{j}}\right) \\
& \leq \max _{k \neq i}\left\{b_{i k}\right\}\left(\rho(A)-a_{i i}\right) \max _{l \neq j}\left\{a_{j l}\right\}\left(\rho(B)-b_{j j}\right) \\
& =t_{i} s_{j}\left(\rho(A)-a_{i i}\right)\left(\rho(B)-b_{j j}\right) .
\end{aligned}
$$

Thus, by solving the quadratic inequality (7), we get that

$$
\begin{aligned}
& \rho(A \circ B) \leq \frac{1}{2}\left\{a_{i i} b_{i i}+a_{j j} b_{j j}+\left[\left(a_{i i} b_{i i}-a_{j j} b_{j j}\right)^{2}+4 t_{i} s_{j}\left(\rho(A)-a_{i i}\right)\left(\rho(B)-b_{j j}\right)\right]^{\frac{1}{2}}\right\} \\
& \leq \max _{i \neq j} \frac{1}{2}\left\{a_{i i} b_{i i}+a_{j j} b_{j j}+\left[\left(a_{i i} b_{i i}-a_{j j} b_{j j}\right)^{2}+4 t_{i} s_{j}\left(\rho(A)-a_{i i}\right)\left(\rho(B)-b_{j j}\right)\right]^{\frac{1}{2}}\right\} .
\end{aligned}
$$

i.e., the conclusion (6) holds.

Case 2. If one of $A$ and $B$ is reducible. We may denote by $P=\left(p_{i j}\right)$ the $n \times n$ permutation matrix with

$$
p_{12}=p_{23}=\cdots=p_{n-1, n}=p_{n, 1}=1 \text {, }
$$

the remaining $p_{i j}$ zero, then both $A+\varepsilon P$ and $B+\varepsilon P$ are irreducible nonnegative matrices for any chosen sufficiently small positive real number $\varepsilon$. Next, we substitute $A+\varepsilon P$ and $B+\varepsilon P$ for $A$ and $B$, respectively, in the previous Case 1 , and then letting $\varepsilon \rightarrow 0$, the result (6) follows by continuity.

Remark 1. Now, we give a comparison between the inequalities (2) and (6). According to the definitions of $t_{i}$ and $s_{j}$, if $t_{i}+b_{i i} \leq \rho(B)$ and $s_{j}+a_{j j} \leq \rho(A)(i, j=1, \cdots, n)$, then $t_{i} s_{j} \leq\left(\rho(B)-b_{i i}\right)\left(\rho(A)-a_{j j}\right)$. Thus, the inequality (6) is better than the inequality (2).

Example 1 ([14]). Let $A$ and $B$ be the following two nonnegative matrices:

$$
A:=\left(a_{i j}\right)=\left(\begin{array}{cccc}
4 & 1 & 0 & 2 \\
1 & 0.05 & 1 & 1 \\
0 & 1 & 4 & 0.5 \\
1 & 0.5 & 0 & 4
\end{array}\right), \quad B:=\left(b_{i j}\right)=\left(\begin{array}{cccc}
1 & 1 & 1 & 1 \\
1 & 1 & 1 & 1 \\
1 & 1 & 1 & 1 \\
1 & 1 & 1 & 1
\end{array}\right) .
$$

By calculation, $\rho(A \circ B)=\rho(A)=5.7339$ and $\rho(B)=4.0$. Thus, the result of Reference [8] (see $p$. 358) is that

$$
\rho(A \circ B) \leq \rho(A) \rho(B)=22.9336
$$


If we respectively apply (1) and (2) to them, according to Reference [14], then

$$
\rho(A \circ B) \leq \max _{1 \leq i \leq 4}\left\{2 a_{i i} b_{i i}+\rho(A) \rho(B)-a_{i i} \rho(B)-b_{i i} \rho(A)\right\}=17.1017,
$$

and

$$
\begin{aligned}
\rho(A \circ B) & \leq \max _{i \neq j} \frac{1}{2}\left\{a_{i i} b_{i i}+a_{j j} b_{j j}+\left[\left(a_{i i} b_{i i}-a_{j j} b_{j j}\right)^{2}\right.\right. \\
& \left.\left.+4\left(\rho(A)-a_{i i}\right)\left(\rho(B)-b_{i i}\right)\left(\rho(A)-a_{j j}\right)\left(\rho(B)-b_{j j}\right)\right]^{\frac{1}{2}}\right\}=11.6478 .
\end{aligned}
$$

However, if Theorem 2 is used, then the following inequality can be obtained:

$$
\begin{aligned}
\rho(A \circ B) \leq & \max _{i \neq j} \frac{1}{2}\left\{a_{i i} b_{i i}+a_{j j} b_{j j}+\left[\left(a_{i i} b_{i i}-a_{j j} b_{j j}\right)^{2}\right.\right. \\
& \left.\left.+4 t_{i} s_{j}\left(\rho(A)-a_{i i}\right)\left(\rho(B)-b_{j j}\right)\right]^{\frac{1}{2}}\right\}=8.1897
\end{aligned}
$$

which shows that the bound in Theorem 2 is the best among the above bounds.

Example 2. Let $A$ and $B$ be the same as the two nonnegative matrices from Reference [18]:

$$
A:=\left(a_{i j}\right)=\left(\begin{array}{ccc}
8 & 7 & 4 \\
0 & 0 & 6 \\
6 & 10 & 7
\end{array}\right), \quad B:=\left(b_{i j}\right)=\left(\begin{array}{ccc}
7 & 6 & 0 \\
3 & 1 & 6 \\
4 & 10 & 7
\end{array}\right) .
$$

By calculation, $\rho(A \circ B)=95.9710, \rho(A)=15.8590$ and $\rho(B)=14.3273$.

According to the result of Reference [8] (see p. 358), we have

$$
\rho(A \circ B) \leq \rho(A) \rho(B)=227.2167 .
$$

If we respectively apply (1) and (2) to them, we get

$$
\rho(A \circ B) \leq \max _{1 \leq i \leq 4}\left\{2 a_{i i} b_{i i}+\rho(A) \rho(B)-a_{i i} \rho(B)-b_{i i} \rho(A)\right\}=211.3577,
$$

and

$$
\begin{aligned}
\rho(A \circ B) & \leq \max _{i \neq j} \frac{1}{2}\left\{a_{i i} b_{i i}+a_{j j} b_{j j}+\left[\left(a_{i i} b_{i i}-a_{j j} b_{j j}\right)^{2}\right.\right. \\
& \left.\left.+4\left(\rho(A)-a_{i i}\right)\left(\rho(B)-b_{i i}\right)\left(\rho(A)-a_{j j}\right)\left(\rho(B)-b_{j j}\right)\right]^{\frac{1}{2}}\right\}=144.1663 .
\end{aligned}
$$

However, if we apply Theorem 2, we can obtain the following inequality:

$$
\begin{aligned}
\rho(A \circ B) \leq & \max _{i \neq j} \frac{1}{2}\left\{a_{i i} b_{i i}+a_{j j} b_{j j}+\left[\left(a_{i i} b_{i i}-a_{j j} b_{j j}\right)^{2}\right.\right. \\
& \left.\left.+4 t_{i} s_{j}\left(\rho(A)-a_{i i}\right)\left(\rho(B)-b_{j j}\right)\right]^{\frac{1}{2}}\right\}=111.5199 .
\end{aligned}
$$

The example also shows that the bound in Theorem 2 is better than the existing bounds.

Corollary 1. If $A$ and $B$ are two stochastic matrices (i.e., probability matrices, transition matrices, or Markov matrices), then

$$
\begin{aligned}
& \rho(A \circ B) \leq \max _{i \neq j} \frac{1}{2}\left\{a_{i i} b_{i i}+a_{j j} b_{j j}+\left[\left(a_{i i} b_{i i}-a_{j j} b_{j j}\right)^{2}+4 t_{i} s_{j}\left(1-a_{i i}\right)\left(1-b_{j j}\right)\right]^{\frac{1}{2}}\right\} \\
& \leq \max _{i \neq j} \frac{1}{2}\left\{a_{i i} b_{i i}+a_{j j} b_{j j}+\left[\left(a_{i i} b_{i i}-a_{j j} b_{j j}\right)^{2}+4\left(1-a_{i i}\right)^{2}\left(1-b_{i i}\right)^{2}\right]^{1 / 2}\right\} .
\end{aligned}
$$

Since the Fan product of two $M$-matrices has a lot of similar properties with the Hadamard product of two nonnegative matrices see References $[3-5,7,8,10,13,14]$. Note that if $A$ is an irreducible 
nonsingular $M$-matrix, then there also exist two positive vectors $u$ and $v$ such that $v^{T} A=\tau(A) v^{T}$ and $A u=\tau(A) u$, respectively. Therefore, we may similarly extend the above result (6) to the case of the Fan product of two $M$-matrices.

Theorem 3. If $A=\left(a_{i j}\right)$ and $B=\left(b_{i j}\right)$ are nonsingular M-matrices, $s_{i}=\max _{j \neq i}\left|a_{i j}\right|, t_{i}=\max _{j \neq i}\left|b_{i j}\right|$, then

$$
\begin{gathered}
\tau(A \star B) \geq \min _{i \neq j} \frac{1}{2}\left\{a_{i i} b_{i i}+a_{j j} b_{j j}-\left[\left(a_{i i} b_{i i}-a_{j j} b_{j j}\right)^{2}\right.\right. \\
\left.\left.+4 t_{i} s_{j}\left(a_{i i}-\tau(A)\right)\left(b_{j j}-\tau(B)\right)\right]^{\frac{1}{2}}\right\} .
\end{gathered}
$$

Proof. This proof is completely similar to that of Theorem 2.

\section{Comparisons of the Simple and Mixed Type Bounds}

Though Theorems 1 and 2 are similar, they are different in form. Next, we give a simple comparison.

Theorem 4. Let $A=\left(a_{i j}\right)$ and $B=\left(b_{i j}\right)$ be two nonnegative matrices, if for any $i \neq j, t_{i} s_{j}(\rho(A)-$ $\left.a_{i i}\right)\left(\rho(B)-b_{j j}\right) \neq t_{j} s_{i}\left(\rho(A)-a_{j j}\right)\left(\rho(B)-b_{i i}\right)$, then Theorem 1 is better than Theorem 2.

Proof. According to (3) and (6), for any $i \neq j$, we need only compare

$$
\max \left\{t_{i} s_{j}\left(\rho(A)-a_{i i}\right)\left(\rho(B)-b_{j j}\right), t_{j} s_{i}\left(\rho(A)-a_{j j}\right)\left(\rho(B)-b_{i i}\right)\right\}:=M_{i j}
$$

with

$$
\min \left\{t_{i} t_{j}\left(\rho(A)-a_{i i}\right)\left(\rho(A)-a_{j j}\right), s_{i} s_{j}\left(\rho(B)-b_{i i}\right)\left(\rho(B)-b_{j j}\right)\right\}:=S_{i j} .
$$

Without loss of generality, let

$$
M_{i j}=t_{i} s_{j}\left(\rho(A)-a_{i i}\right)\left(\rho(B)-b_{j j}\right) .
$$

We assume that $M_{i j}<S_{i j}$, i.e.,

$t_{i} s_{j}\left(\rho(A)-a_{i i}\right)\left(\rho(B)-b_{j j}\right)<\min \left\{t_{i} t_{j}\left(\rho(A)-a_{i i}\right)\left(\rho(A)-a_{j j}\right), s_{i} s_{j}\left(\rho(B)-b_{i i}\right)\left(\rho(B)-b_{j j}\right)\right\}$.

Then,

$$
0 \leq s_{j}\left(\rho(B)-b_{j j}\right)<t_{j}\left(\rho(A)-a_{j j}\right)
$$

and

$$
0 \leq t_{i}\left(\rho(A)-a_{i i}\right)<s_{i}\left(\rho(B)-b_{i i}\right)
$$

Therefore,

$$
0 \leq t_{i} s_{j}\left(\rho(A)-a_{i i}\right)\left(\rho(B)-b_{j j}\right)<s_{i} t_{j}\left(\rho(B)-b_{i i}\right)\left(\rho(A)-a_{j j}\right),
$$

which is in conflict with the previous condition (9). Thus, we see that $M_{i j} \geq S_{i j}$ for any $i \neq j$. This proof is completed.

From the above discussion, we see that, generally speaking, $M_{i j} \geq S_{i j}$ for any $i \neq j$ when $t_{i} s_{j}\left(\rho(A)-a_{i i}\right)\left(\rho(B)-b_{j j}\right) \neq s_{i} t_{j}\left(\rho(B)-b_{i i}\right)\left(\rho(A)-a_{j j}\right)$. Next, let us consider the case of $t_{i} s_{j}(\rho(A)-$ $\left.a_{i i}\right)\left(\rho(B)-b_{j j}\right)=s_{i} t_{j}\left(\rho(B)-b_{i i}\right)\left(\rho(A)-a_{j j}\right)$.

Theorem 5. Let $A=\left(a_{i j}\right)$ and $B=\left(b_{i j}\right)$ be two nonnegative matrices, if for any $i \neq j, t_{i} s_{j}(\rho(A)-$ $\left.a_{i i}\right)\left(\rho(B)-b_{j j}\right)=t_{j} s_{i}\left(\rho(A)-a_{j j}\right)\left(\rho(B)-b_{i i}\right)$ and $\frac{t_{j}}{s_{j}}=\frac{\rho(B)-b_{j j}}{\rho(A)-a_{j j}}$, then Theorem 2 is equivalent to Theorem 1. 
Proof. According to the above Theorem 4, let us reconsider the relationship between $M_{i j}$ and $S_{i j}$ for any $i \neq j$. For convenience, we define

$$
\alpha_{j}=\frac{s_{j}\left(\rho(B)-b_{j j}\right)}{t_{j}\left(\rho(A)-a_{j j}\right)}
$$

Obviously,

$$
t_{i} t_{j}\left(\rho(A)-a_{i i}\right)\left(\rho(A)-a_{j j}\right)=s_{i} t_{j}\left(\rho(B)-b_{i i}\right)\left(\rho(A)-a_{j j}\right) \frac{1}{\alpha_{j}},
$$

and

$$
s_{i} s_{j}\left(\rho(B)-b_{i i}\right)\left(\rho(B)-b_{j j}\right)=t_{i} s_{j}\left(\rho(B)-b_{j j}\right)\left(\rho(A)-a_{i i}\right) \alpha_{j} .
$$

Therefore, if $\alpha_{j} \neq 1$, then we have always $M_{i j}>S_{i j}$ for any $i \neq j$ under the conditions of this theorem. However, when $\alpha_{j}=1$, i.e., $\frac{t_{j}}{s_{j}}=\frac{\rho(B)-b_{j j}}{\rho(A)-a_{j j}}$, we have $M_{i j}=S_{i j}$ for any $i \neq j$. Therefore, Theorem 2 is equivalent to Theorem 1 . Thus, the proof is completed.

\section{Inequalities for the Fan Product of Several M-Matrices}

In the previous sections, we mainly consider the Hadamard product of two matrices. In fact, there also exist many of similar inequalities for the minimum eigenvalue of Fan product of two $M$-matrices:

- In Reference [8], p. 359, Horn and Johnson pointed out that

$$
\tau(A \star B) \geq \tau(A) \tau(B) .
$$

- In 2007, Fang gave another lower bound in the Remark 3 of Reference [4]:

$$
\tau(A \star B) \geq \min _{1 \leq i \leq n}\left\{b_{i i} \tau(A)+a_{i i} \tau(B)-\tau(A) \tau(B)\right\} .
$$

- In 2009, Liu et al. [14] gave a sharper bound than (14), i.e.,

$$
\begin{aligned}
\tau(A \star B) & \geq \frac{1}{2} \min _{i \neq j}\left\{a_{i i} b_{i i}+a_{j j} b_{j j}-\left[\left(a_{i i} b_{i i}-a_{j j} b_{j j}\right)^{2}\right.\right. \\
& \left.\left.+4\left(b_{i i}-\tau(B)\right)\left(a_{i i}-\tau(A)\right)\left(b_{j j}-\tau(B)\right)\left(a_{j j}-\tau(A)\right)\right]^{\frac{1}{2}}\right\} .
\end{aligned}
$$

Note that the classes of $M$-matrices and $H$-matrices are both closed under the Fan product (see Observation 5.7.2 in Reference [8]). Therefore, we may consider the case of the product of several matrices. For convenience, we shall continue to use the notation employed previously. However, according to Reference [8], the definition of the function $\tau(\cdot)$ should be extended to general matrices via the comparison matrix. The comparison matrix $M(A)=\left(m_{i j}\right)$ of a given matrix $A=\left(a_{i j}\right) \in \mathbb{C}^{n \times n}$ is defined by

$$
m_{i j}= \begin{cases}-\left|a_{i j}\right|, & \text { if } i \neq j, \\ \left|a_{i i}\right|, & \text { if } i=j .\end{cases}
$$

Definition 1 ([8]). For any $A \in \mathbb{R}^{n \times n}, \tau(A):=\tau(M(A))$, where $M(A)$ is the comparison matrix of $A$.

In addition, if $A=\left(a_{i, j}\right) \in \mathbb{R}^{m \times n}$ has nonnegative entries and $\alpha \geq 0$, we write $A^{(\alpha)} \equiv\left(a_{i, j}^{\alpha}\right)$ for the $\alpha$ th Hadamard power of $A$. Moreover, we use the convention $0^{0} \equiv 0$ to ensure continuity in $a$ for $\alpha \geq 0$, see Reference [8]. 
In Reference [8], it is shown by Theorem 5.7.15 that if $A_{1}, \ldots A_{m}$ are $n \times n H$-matrices and $\alpha_{1}, \alpha_{2}, \ldots, \alpha_{m} \geq 0$ satisfy $\sum_{k=1}^{m} \alpha_{k} \geq 1$, then

$$
\tau\left(A_{1}^{\left(\alpha_{1}\right)} \circ \cdots \circ A_{m}^{\left(\alpha_{m}\right)}\right) \geq \prod_{k=1}^{m}\left[\tau\left(A_{k}\right)\right]^{\alpha_{k}},
$$

where $A^{(\alpha)}$ is again defined as entrywise and any scalar definition of $a^{\alpha}$ such that $\left|a^{\alpha}\right|=|a|^{\alpha}$ is allowed (see Reference [8]). Next, for convenience, we define

$$
a^{\alpha}:= \begin{cases}a^{\alpha}, & \text { if } a \geq 0, \\ -|a|^{\alpha}, & \text { if } a<0 .\end{cases}
$$

The above theorem (16) provides a beautiful result, which encourages us to continue researching this problem. Since for any $H$-matrix $A$, according to Definition $1, \tau(A)=\tau(M(A))$. Therefore, we need only consider the $M$-matrix case.

First, let us recall the following lemmas:

Lemma 4 ([12]). Let $A$ be an irreducible nonsingular M-matrix, if $A Z \geq k Z$ for a nonnegative nonzero vector $Z$, then $k \leq \tau(A)$.

Lemma 5 ([19]). Let $x_{j}=\left(x_{j}(1), \cdots, x_{j}(n)\right)^{T} \geq 0, j \in\{1,2, \cdots, m\}$, if $P_{j}>0$ and $\sum_{k=1}^{m} \frac{1}{P_{k}} \geq 1$, then we have

$$
\sum_{i=1}^{n} \prod_{j=1}^{m} x_{j}(i) \leq \prod_{j=1}^{m}\left\{\sum_{i=1}^{n}\left[x_{j}(i)\right]^{P_{j}}\right\}^{\frac{1}{P_{j}}} .
$$

Next, according to these lemmas, we generalize the inequality (14) of the Fan product of two matrices to the Fan product of several matrices.

Theorem 6. For any positive integer $P_{k}$ with $\sum_{k=1}^{m} \frac{1}{P_{k}} \geq 1$, if $A_{k} \in M_{n}$ for all $k \in\{1,2, \cdots, m\}$, then

$$
\tau\left(A_{1} \star A_{2} \cdots \star A_{m}\right) \geq \min _{1 \leq i \leq n}\left\{\prod_{k=1}^{m} A_{k}(i, i)-\prod_{k=1}^{m}\left[A_{k}(i, i)^{P_{k}}-\tau\left(A_{k}^{\left(P_{k}\right)}\right)\right]^{\frac{1}{P_{k}}}\right\} .
$$

Proof. It is quite evident that (19) holds with the equality for $n=1$. Below, we assume that $n \geq 2$.

Case 1. Suppose that $A_{k}(k \in\{1,2, \cdots, m\})$ is irreducible, then $A_{k}^{\left(P_{k}\right)}$ is also irreducible. Let $u_{k}^{\left(P_{k}\right)}=\left(u_{k}(1)^{P_{k}}, \cdots, u_{k}(n)^{P_{k}}\right)^{T}>0$ be a right Perron eigenvector of $A_{k}^{\left(P_{k}\right)}$, and $u_{k}=$ $\left(u_{k}(1), \cdots, u_{k}(n)\right)^{T}>0$, thus for any $i \in N$, we have

$$
\begin{gathered}
A_{k}^{\left(P_{k}\right)} u_{k}^{\left(P_{k}\right)}=\tau\left(A_{k}^{\left(P_{k}\right)}\right) u_{k}^{\left(P_{k}\right)}, \\
A_{k}(i, i)^{P_{k}} u_{k}(i)^{P_{k}}-\sum_{j \neq i}\left|A_{k}(i, j)^{P_{k}}\right| u_{k}(j)^{P_{k}}=\tau\left(A_{k}^{\left(P_{k}\right)}\right) u_{k}(i)^{\left(P_{k}\right)},
\end{gathered}
$$

i.e.,

$$
\sum_{j \neq i}\left|A_{k}(i, j)^{P_{k}}\right| u_{k}(j)^{P_{k}}=\left(A_{k}(i, i)^{P_{k}}-\tau\left(A_{k}^{\left(P_{k}\right)}\right)\right) u_{k}(i)^{P_{k}}
$$


Denote $C=A_{1} \star A_{2} \cdots \star A_{m}, Z=u_{1} \star u_{2} \cdots \star u_{m}=(Z(1), \cdots, Z(n))^{T}>0$, where $Z(i)=$ $\prod_{k=1}^{m} u_{k}(i)$. By Lemma 5 and (20), we get that

$$
\begin{aligned}
(C Z)_{i} & =\left(\prod_{k=1}^{m} A_{k}(i, i)\right) Z(i)-\left(\sum_{j \neq i} \prod_{k=1}^{m}\left|A_{k}(i, j)\right|\right) Z(j) \\
& =\left(\prod_{k=1}^{m} A_{k}(i, i)\right) Z(i)-\sum_{j \neq i} \prod_{k=1}^{m}\left(\left|A_{k}(i, j)\right| u_{k}(j)\right) \\
& \geq\left(\prod_{k=1}^{m} A_{k}(i, i)\right) Z(i)-\prod_{k=1}^{m}\left\{\sum_{j \neq i}\left[\left|A_{k}(i, j)\right| u_{k}(j)\right]^{\left(P_{k}\right)}\right\}^{\frac{1}{P_{k}}} \text { (by the equality (20)) } \\
& =\left(\prod_{k=1}^{m} A_{k}(i, i)\right) Z(i)-\prod_{k=1}^{m}\left\{\left[A_{k}(i, i)^{P_{k}}-\tau\left(A_{k}^{\left(P_{k}\right)}\right)\right] u_{k}(i)^{P_{k}}\right\}^{\frac{1}{P_{k}}} \\
& =\left\{\prod_{k=1}^{m} A_{k}(i, i)-\prod_{k=1}^{m}\left[A_{k}(i, i)^{P_{k}}-\tau\left(A_{k}^{\left(P_{k}\right)}\right)\right]\right\}^{\frac{1}{P_{k}}} Z(i) .
\end{aligned}
$$

According to the Lemma 4, we obtain that

$$
\tau\left(A_{1} \star A_{2} \cdots \star A_{m}\right) \geq \min _{1 \leq i \leq n}\left\{\prod_{k=1}^{m} A_{k}(i, i)-\prod_{k=1}^{m}\left[A_{k}(i, i)^{P_{k}}-\tau\left(A_{k}^{\left(P_{k}\right)}\right)\right]^{\frac{1}{P_{k}}}\right\} .
$$

Case 2. If one of $A_{k}(i=1,2, \cdots, m)$ is reducible. Similar to Case 2 of the previous Theorem 2, let $P=\left(p_{i j}\right)$ be the $n \times n$ permutation matrix with $p_{12}=p_{23}=\cdots=p_{n-1, n}=p_{n, 1}=1$, the remaining $p_{i j}$ zero, then $A_{k}-\varepsilon P$ is an irreducible nonsingular $M$-matrix for any chosen positive real number $\varepsilon$. Now we substitute $A_{k}-\varepsilon P$ for $A_{k}$, in the previous Case 1, and then letting $\varepsilon \rightarrow 0$, the result (19) follows by continuity.

Remark 2. If we take $m=2$ in Theorem 6, one can obtain the following results:

- If $p_{1}=p_{2}=1, A_{1}=A=\left(a_{i j}\right), A_{2}=B=\left(b_{i j}\right)$, we have

$$
\tau(A \star B) \geq \min _{1 \leq i \leq n}\left\{a_{i i} b_{i i}-\left(a_{i i}-\tau(A)\right)\left(b_{i i}-\tau(B)\right)\right\},
$$

which is just the inequality (14).

- If $p_{1}=p_{2}=2, A_{1}=A=\left(a_{i j}\right), A_{2}=B=\left(b_{i j}\right)$, then

$$
\tau(A \star B) \geq \min _{1 \leq i \leq n}\left\{a_{i i} b_{i i}-\left[a_{i i}^{2}-\tau(A \star A)\right]^{\frac{1}{2}}\left[b_{i i}^{2}-\tau(B \star B)\right]^{\frac{1}{2}}\right\} .
$$

In addition, by using the inequalities of arithmetic and geometric means, we know that

$$
a_{i i}^{2} \tau(B \star B)+b_{i i}^{2} \tau(A \star A) \geq 2 a_{i i} b_{i i}[\tau(A \star A) \tau(B \star B)]^{\frac{1}{2}},
$$

so

$$
\left(a_{i i}^{2}-\tau(A \star A)\right)\left(b_{i i}^{2}-\tau(B \star B)\right) \leq\left\{a_{i i} b_{i i}-[\tau(A \star A) \tau(B \star B)]^{\frac{1}{2}}\right\}^{2} .
$$

Since for any $A, B \in M_{n}, \tau(A \star B) \geq \tau(A) \tau(B)$ (see Reference [14] or (13)), then, by (22), we know that

$$
a_{i i} b_{i i}-\left[\left(a_{i i}^{2}-\tau(A \star A)\right)\left(b_{i i}^{2}-\tau(B \star B)\right)\right]^{\frac{1}{2}} \geq[\tau(A \star A) \tau(B \star B)]^{\frac{1}{2}} \geq \tau(A) \tau(B) .
$$

Therefore, the inequality (21) is better than the inequality (13). In addition, the following example shows that the inequality (21) is also better than the inequality (15). 
Example 3. ([14]). Consider the following two $3 \times 3$ M-matrices:

$$
A:=\left(a_{i j}\right)=\left(\begin{array}{ccc}
2 & -1 & 0 \\
0 & 1 & -0.5 \\
-0.5 & -1 & 2
\end{array}\right), \quad B:=\left(b_{i j}\right)=\left(\begin{array}{ccc}
1 & -0.25 & -0.25 \\
-0.5 & 1 & -0.25 \\
-0.25 & -0.5 & 1
\end{array}\right) .
$$

By direct calculation, $\tau(A)=0.5402, \tau(B)=0.3432$ and $\tau(A \star B)=0.9377$. According to Reference [14], the inequality (15) shows that

$$
\tau(A \star B) \geq 0.7655
$$

If we apply (21) to them, then

$$
\tau(A \star B) \geq \min _{1 \leq i \leq n}\left\{a_{i i} b_{i i}-\left[a_{i i}^{2}-\tau(A \star A)\right]^{\frac{1}{2}}\left[b_{i i}^{2}-\tau(B \star B)\right]^{\frac{1}{2}}\right\}=0.8579,
$$

which shows that our result is closer to the exact value 0.9377.

- If $p_{1}=1, p_{2}=2, A_{1}=A=\left(a_{i j}\right), A_{2}=B=\left(b_{i j}\right)$, then we get

$$
\tau(A \star B) \geq \min _{1 \leq i \leq n}\left\{a_{i i} b_{i i}-\left[a_{i i}-\tau(A)\right]\left[b_{i i}^{2}-\tau(B \star B)\right]^{\frac{1}{2}}\right\} .
$$

\section{Concluding Remarks}

This paper mainly presents the relationship between the simple type [3] and the mixed type (6), which perfects the corresponding theory. In addition, we also research the problem on the minimum eigenvalue of the Fan product of more $M$-matrices and obtain several interesting results, see the inequalities (19), (22), and (23). Since for any $A \in \mathcal{M}_{n}, \tau(A \star A) \geq(\tau(A))^{2}$, numerical examples and some analyses show that the special cases (e.g., (22) and (23)) of the inequality (19) improve some known results stated in this paper.

Finally, it is worth mentioning that there also exist other products in statistics or econometrics, such as the block Hadamard product [20], Khatri-Rao and Tracy-Singh products [21]. Are there similar results regarding these products? This may still be an interesting problem.

Author Contributions: Conceptualization, Q.G.; Funding acquisition, J.L. and H.L.; Date, Q.G.; Supervision, J.L., H.L. and C.C.; Writing—original draft, Q.G.; and Writing—review and editing, J.L., H.L. and C.C.

Funding: This research was funded by the Natural Science Foundation of China $(11271001,61370147,11101071)$, the Fundamental Research Funds for the Central Universities (ZYGX2016J138), and Sichuan Province Science and Technology Research Project (12ZC1802).

Acknowledgments: The authors would like to thank reviewers and editors for providing us some good suggestions and help.

Conflicts of Interest: The authors declare no conflict of interest.

\section{References}

1. Magnus, J.R.; Neudecker, H. Matrix Differential Calculus with Applications in Statistics and Econometrics; Wiley: Chichester, UK, 1988.

2. Styan, G.P.H. Hadamard products and multivariate statistical analysis. Linear Algebra Appl. 1973, 6, $217-240$. [CrossRef]

3. Cheng, G.H. New bounds for eigenvalues of the Hadamard product and the Fan product of matrices. Tanwan J. Math. 2014, 18, 305-312. [CrossRef]

4. Fang, M.Z. Bounds on the eigenvalus of the Hadamard product and the Fan product of matrices. Linear Algebra Appl. 2007, 425, 7-15. [CrossRef] 
5. Chen, F.B. Some new inequalities for the Hadamard product of M-matrices. J. Inequal. Appl. 2013, $2013,581$. [CrossRef]

6. Chen, S.C. A lower bound for the minimum eigenvalue of the Hadamard product of matrices. Linear Algebra Appl. 2004, 378, 159-166. [CrossRef]

7. Guo, Q.P.; Li, H.B.; Song, M.Y. New inequalities on eigenvalues of the Hadamard product and the Fan product of matrices. J. Inequal. Appl. 2013, 2013, 433. [CrossRef]

8. Horn, R.A.; Johnson, C.R. Topics in Matrix Analysis; Cambridge University Press: Cambridge, UK; New York, NY, USA, 1991.

9. Guo, Q.P.; Leng, J.S.; Han, D.G.; Fan, Q.; Li, H.; Gao, Q. Adaptive Optimal Dual Frames for Signal Reconstruction with Erasures. IEEE Access 2016, 4, 7577-7584. [CrossRef]

10. Huang, R. Some inequalities for the Hadamard product and the Fan product of matrices. Linear Algebra Appl. 2008, 428, 1551-1559. [CrossRef]

11. Li, D.W.; Leng, J.S.; Huang, T.Z.; Xu, Y.X. Some equalities and inequalities for probabilistic frames. J. Inequal. Appl. 2016, 2016, 245. [CrossRef]

12. Li, H.B.; Huang, T.Z.; Shen, S.Q.; Li, H. Lower bounds for the minimum eigenvalue of Hadamard product of an M-matrix and its inverse. Linear Algebra Appl. 2007, 420, 235-247. [CrossRef]

13. Li, Y.T.; Li, Y.Y.; Wang, R.W.; Wang, Y.Q. Some new bounds on eigenvalues of the Hadamard product and the Fan product of matrices. Linear Algebra Appl. 2010, 432, 536-545. [CrossRef]

14. Liu, Q.B.; Chen, G.L. On two inequalities for the Hadamard product and the Fan product of matrices. Linear Algebra Appl. 2009, 431, 974-984. [CrossRef]

15. Visick, G. A quantitative version of the observation that the Hadamard product is a principal submatrix of the Kronecker. Linear Algebra Appl. 2000, 304, 45-68. [CrossRef]

16. Zhang, Y. Some spectral norm inequalities on Hadamard products of nonnegative matrices. Linear Algebra Appl. 2018, 556, 162-170. [CrossRef]

17. Plemmons, R.J.; Berman, A. Nonnegative Matrices in the Mathematical Sciences; SIAM Press: Philadelphia, PA, USA, 1979.

18. Huang, Z. On the spectral radius and the spectral norm of Hadamard products of nonnegative matrices. Linear Algebra Appl. 2010, 434, 457-462. [CrossRef]

19. Hardy, G.H.; Littlewood, J.E.; Polya, G. Inequality, 2nd ed.; Cambridge University Press: Cambridge, UK; New York, NY, USA, 1988.

20. Li, M.H. An Oppenheim type inequality for a block Hadamard product. Linear Algebra Appl. 2014, 452, 1-6.

21. Liu, S.Z. Matrix results on the Khatri-Rao and Tracy-Singh products. Linear Algebra Appl. 1999, 289, $267-277$. [CrossRef]

(C) 2019 by the authors. Licensee MDPI, Basel, Switzerland. This article is an open access article distributed under the terms and conditions of the Creative Commons Attribution (CC BY) license (http://creativecommons.org/licenses/by/4.0/). 